\title{
Honokiol Suppresses Renal Cancer Cells' Metastasis via Dual-Blocking Epithelial-Mesenchymal Transition and Cancer Stem Cell Properties through Modulating miR-141/ZEB2 Signaling
}

\author{
Weidong $\mathrm{Li}^{1,5}$, Qian Wang ${ }^{2,5}$, Qiaozhen $\mathrm{Su}^{3,5}$, Dandan $\mathrm{Ma}^{4}$, Chang $\mathrm{An}^{3}$, Lei $\mathrm{Ma}^{1, \star}$, and \\ Hongfeng Liang ${ }^{3, *}$
}

Renal cell carcinoma (RCC) is associated with a high frequency of metastasis and only few therapies substantially prolong survival. Honokiol, isolated from Magnolia spp. bark, has been shown to exhibit pleiotropic anticancer effects in many cancer types. However, whether honokiol could suppress RCC metastasis has not been fully elucidated. In the present study, we found that honokiol suppressed renal cancer cells' metastasis via dual-blocking epithelial-mesenchymal transition (EMT) and cancer stem cell (CSC) properties. In addition, honokiol inhibited tumor growth in vivo. It was found that honokiol could upregulate miR-141, which targeted ZEB2 and modulated ZEB2 expression. Honokiol reversed EMT and suppressed CSC properties partly through the miR-141/ZEB2 axis. Our study suggested that honokiol may be a suitable therapeutic strategy for RCC treatment.

\section{INTRODUCTION}

Renal cell carcinoma (RCC), accounting for $90 \%$ to $95 \%$ of all renal tumors in adults, comprises a heterogeneous group of epithelial neoplasms with diverse biological potential and variable clinical outcomes (McLaughlin et al., 2006). Patients with advanced RCC have poor prognosis and high mortality, even after treatment (Motzer et al., 1999). Moreover, multi-drug resis-

${ }^{1}$ Department of Medical Oncology, Affiliated Cancer Hospital of Guangzhou Medical University, Cancer Center of Guangzhou Medical University (CCGMU), ${ }^{2}$ The Medical Faculty of Jinan University, ${ }^{3}$ Department of Neurology, the Second Affiliated Hospital of Guangzhou University of Chinese Medicine, ${ }^{4}$ Internal Medicine of Traditional Chinese Medicine, Guangzhou University of Chinese Medicine, Guangzhou, People's Republic of China, ${ }^{5}$ These authors contributed equally to this work. *Correspondence: leima01@yeah.net (LM); hongfengliang@yeah.net $(\mathrm{HL})$

Received 20 January, 2014; revised 30 March, 2014; accepted 31 March, 2014; published online 8 May, 2014

Keywords: cancer stem cell, epithelial-mesenchymal transition, honokiol, microRNA, renal cell carcinoma tance is a difficult problem faced in the treatment of advanced RCC (Cheng et al., 2009). Thus, there is an urgent need to develop a more effective therapeutic agent.

Mounting evidence suggests that epithelial-mesenchymal transition (EMT) plays a significant role in cancer invasion and metastasis through the formation of cells with a more motile and invasive phenotype (Polyak and Weinberg, 2009). Moreover, the induction of EMT has been shown to result in the enrichment of cells with stem-like properties, termed cancer stem cells (CSCs), which are believed to be the origin of cancer progression, distant metastasis and drug resistance (Mani et al., 2008; Radisky and LaBarge, 2008). Key factors controlling EMT and CSCs have been identified as the potential targets for the prevention and treatment of metastatic cancers (Gupta et al., 2009). MicroRNAs (miRNAs), a class of evolutionary conserved small non-coding RNAs (18-22 nucleotides) that inhibit gene expression (Ambros, 2004), are suggested to regulate gene networks involved in EMT and CSCs (Croce and Calin, 2005; Gregory et al., 2008; Taube et al., 2013).

In recent decades, extracts from natural products have been investigated for the treatment of many malignant tumors. Newman et al. (2003) reported that more than half of the new chemicals approved between 1982 and 2002 were derived directly or indirectly from natural products. Honokiol is one of two dominant biphenolic compounds isolated from Magnolia spp. bark, which has been extensively used in traditional Chinese medicine (Fried and Arbiser, 2009; Lee et al., 2011). Preclinical research on honokiol's broad-ranging capabilities shows its potential as a therapeutic compound for numerous solid and hematological cancers, including its effectiveness in combating multi-drug resistance and its synergy with other anticancer therapies (Kumar et al., 2013; Tian et al., 2012). However, it is unknown whether honokiol exerts anti-tumor effects through inhibiting EMT and CSCs in RCC. In this study, we examine the effects of honokiol on RCC through in vitro and in vivo experiments.

\section{MATERIALS AND METHODS}

Cell culture and honokiol treatment

A-498 cell line was maintained in Dulbecco's modified Eagle's 
medium (DMEM, Gibco, USA) supplemented with $10 \%$ fetal bovine serum (FBS) at $37^{\circ} \mathrm{C}$ with $5 \% \mathrm{CO}_{2}$ in a humidified incubator. Honokiol was purchased from Sigma (USA) and dissolved in dimethylsulfoxide (DMSO). For honokiol treatment, A498 cells were incubated with appropriate concentrations of honokiol $(2.5,5,10,20,40,80 \mu \mathrm{mol})$. DMSO solution without honokiol was used as a control.

Plasmids and cell transfection

MiR-141 inhibitor was synthesized by Genepharma (China). Full length ZEB2 cDNA was purchased from GeneCopeia (USA) and subcloned into the eukaryotic expression vector pcDNA.3 (Invitrogen). Transfection was performed with Lipofectamine 2000 reagent (Invitrogen).

\section{Luciferase reporter assay}

For the reporter gene assay, cells seeded in 24-well plates were transfected with $200 \mathrm{ng}$ ZEB2-luc and $1 \mathrm{ng}$ of the pRLSV40 Renilla luciferase construct (as an internal control) for 24 $\mathrm{h}$, and then subjected to honokiol. Cell extracts were prepared $48 \mathrm{~h}$ after treatment, and the luciferase activity was measured using the Dual-Luciferase Reporter Assay System (Promega).

RNA extraction and quantitative RT-PCR

Total RNA was extracted with TRIzol reagent according to the manufacturer's instructions (Invitrogen, USA). cDNA was synthesized with the PrimeScript RTreagent Kit (Promega, USA). The primer sequences of ZEB2 were: $5^{\prime}$-TCTCGCCCGAGTG AAGCCTT-3' (Forward); 5'-GGGAGAATTGCTTGATGGAGC3' (Reverse).

\section{Cell migration and invasion assays}

The cell migration capabilities in vitro were determined using a Transwell assay as described previously (Moutasim et al., 2011). A transwell chamber coated with Matrigel was used to determine the cell invasion capabilities in vitro as described previously (Valster et al., 2005).

MTT and colony formation assays

The inhibitory effect of honokiol on RCC cell viability was evaluated by using a 3-(4, 5-dimethylthiazol-2-yl)-2, 5-diphenyl tetrazolium bromide (MTT) assay (MTT; Sigma). Cells were seeded at a density of 1500 cells per well in 96-well culture plates and treated with increasing concentrations of honokiol as indicated in Fig. 1A. After $72 \mathrm{~h}$ of incubation, $40 \mu \mathrm{l}$ of MTT at 5 $\mathrm{mg} / \mathrm{ml}$ was added to each well, and incubation was continued for $2 \mathrm{~h}$. The formazan crystals, resulting from the mitochondrial enzymatic activity on the MTT substrate, were solubilized with $100 \mu \mathrm{l}$ of DMSO. Absorbance was measured at $590 \mathrm{~nm}$ using a microplate reader.

For colony formation assay, cells treated with honokiol or DMSO were seeded in 6-well culture plates and cultured for two weeks. The colonies obtained were formalin fixed and stained with hematoxylin.

Sphere formation and Hoechst 33342 exclusion assay Tumor sphere formation assay was carried out according to our previous study (Ma et al., 2013). Briefly, single cells were plated in Ultra Low Attachment plates (Corning) in serum-free DMEMF12 supplemented with $10 \mathrm{ng} / \mathrm{ml} \mathrm{bFGF,} 10 \mathrm{ng} / \mathrm{ml} \mathrm{EGF}$, and B27 (all from invitrogen). In these conditions cells grew as spherical clusters in suspension. The numbers of spheres with a diameter over $50 \mu \mathrm{m}$ were counted under a microscope.

For the Hoechst 33342 exclusion assay, cells were incubated with Hoechst $33342(5 \mu \mathrm{g} / \mathrm{ml}$, Invitrogen) in medium containing
$5 \%$ FBS at $37^{\circ} \mathrm{C}$ for 90 min. Following this incubation, cells were washed with ice-cold PBS, stained with propidium iodide $(1 \mu \mathrm{g} / \mathrm{ml})$, and maintained at $4^{\circ} \mathrm{C}$ for flow cytometry analyses using a FACSAria Flow cytometer (Beckton Dickson).

\section{Quantitative RT-PCR}

Total RNA from cultured cells was extracted using the TRIzol reagent (Invitrogen, USA). Real-time PCR was carried out using an $\mathrm{ABI} 7900 \mathrm{HT}$ fast real-time PCR system (Applied Biosystems, USA). The following primers were used: E-cadherin, 5'CCCACCACGTACAAGGGTC-3' (sense), 5'-ATGCCATCGTT GTTCACTGGA-3' (antisense); ZEB2, 5'-CCGCCCCTGAAGG TTATTCC-3' (sense) and 5'-TGCCTGATTCATTCTGCTAACA A-3' (antisense); Vimentin, 5'-ACCGCTTTGCCAACTACAT-3' (s ense) and 5'-TTGTCCCGCTCCACCTC-3' (antisense); Fibronectin, 5'-CCATCGCAAACCGCTGCCAT-3' (sense) and 5'AACACTTCTCAGCTATGGGCTT-3' (antisense); GAPDH, 5'ACCACAGTCCATGCCATCAC-3' (sense), and 5'-TCCACCAC CCTGTTGCTGTA-3' (antisense)

Western blot analysis and Immunohistochemical (IHC) staining

Cells were treated with honokiol for $48 \mathrm{~h}$, and then total cellular protein lysates were prepared with RIPA buffer $[50 \mathrm{mM}$ Tris $(\mathrm{pH}$ 8.0), $150 \mathrm{mM} \mathrm{NaCl}, 0.1 \%$ SDS, $1 \% \mathrm{NP} 40$ and $0.5 \%$ sodium deoxycholate] containing proteinase inhibitors, $1 \%$ Cocktail and $1 \mathrm{mM}$ PMSF (Sigma, USA). A total of $20 \mu \mathrm{g}$ protein sample was separated by $10 \%$ SDS-PAGE and transferred to nitrocellulose membranes. After blocking the membranes with $3 \%$ BSA in Tris-buffered saline with $0.1 \%$ Tween 20 (pH 7.6, TBST), the membranes were incubated with primary antibodies at $4^{\circ} \mathrm{C}$ overnight. After being washed with TBST, the membranes were incubated with secondary antibodies at room temperature for 1 $h$ and visualized with an ECL chemiluminescent detection system (Pierce, USA). GAPDH protein levels were used for normalization. The primary antibodies E-cadherin, Vimentin, fibronectin, ZEB2 and GAPDH were from Santa Cruz Biotechnology, Inc (USA).

For immunohistochemistry, paraffin-embedded sections were deparaffinized in xylene and rehydrated in graded alcohol. Antigen retrieval was done by boiling the slides in $10 \mathrm{mM}$ sodium citrate buffer, $\mathrm{pH}$ 6.0. Staining was done using the EliVision Plus Kit (Maixin Bio, China) according to the manufacturer's protocol. DAB was used as a substrate for peroxidase.

Xenograft tumor mouse model

In the tumor growth experiment, A-498 cells treated with control (DMSO) or honokiol were subcutaneously injected into BALB/c nude mice $\left(4 \times 10^{6}\right.$ cellsper mouse). The tumor sizes were measured by vernier caliper and calculated using the formula $\mathrm{V}$ $=\left(\mathrm{LW}^{2}\right) \pi / 6\left[\mathrm{~V}\right.$, volume $\left(\mathrm{mm}^{3}\right) ; \mathrm{L}$, biggest diameter $(\mathrm{mm}) ; \mathrm{W}$, smallest diameter $(\mathrm{mm})]$.

Statistical analysis

All assays were repeated in triplicates in three independent experiments, and quantitative data were presented as mean \pm SD. The differences between two groups were compared by the 2-tailed Student's $t$-test. A $P$ value of $<0.05$ was considered statistically significant. All statistical analyses were performed using SPSS 15.0 (SPSS Inc., USA).

\section{RESULTS}

Honokiol suppresses proliferation of RCC cells in vitro We analyzed cell viability by MTT assay to evaluate the effect 
A

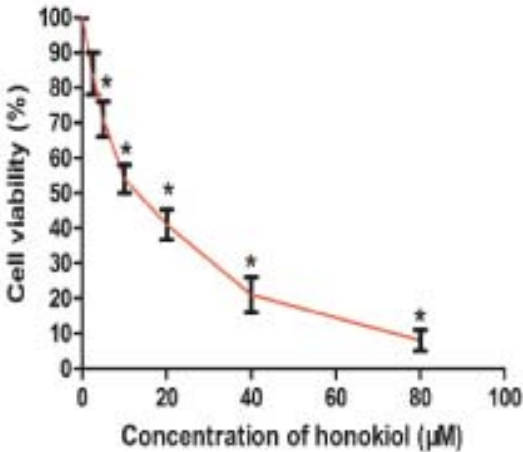

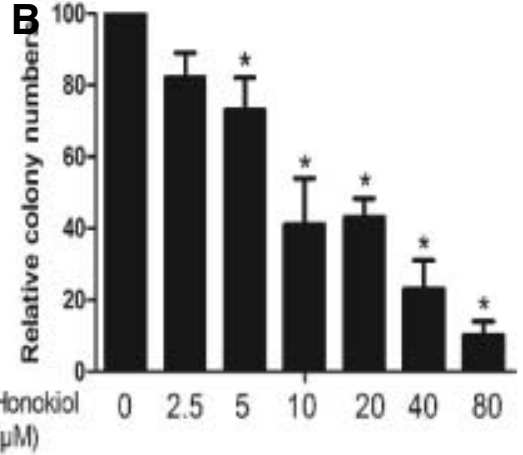

Fig. 1. Antiproliferative effects of honokiol in RCC cells. (A) A498 cells were treated with various concentrations of honokiol and cell viability was determined by MTT assay. (B) The colony formation assay showed that honokiol impaired A-498 cells' ability to form colonies.

A
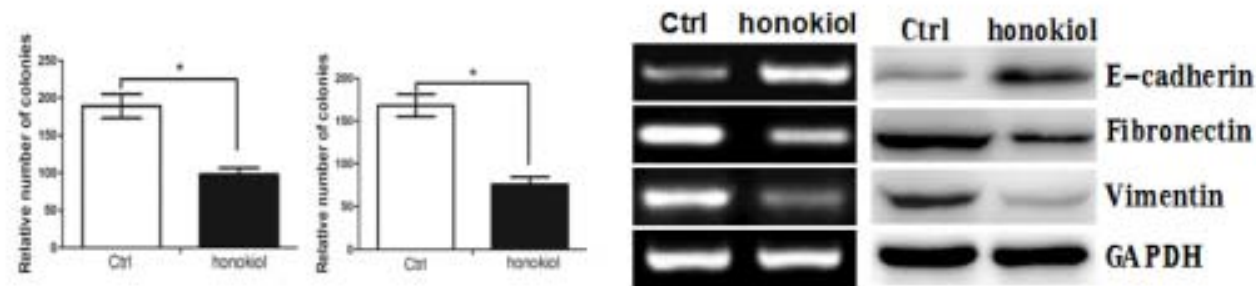

B

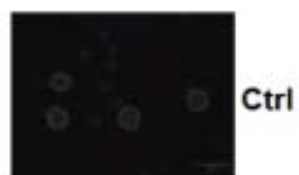

trl
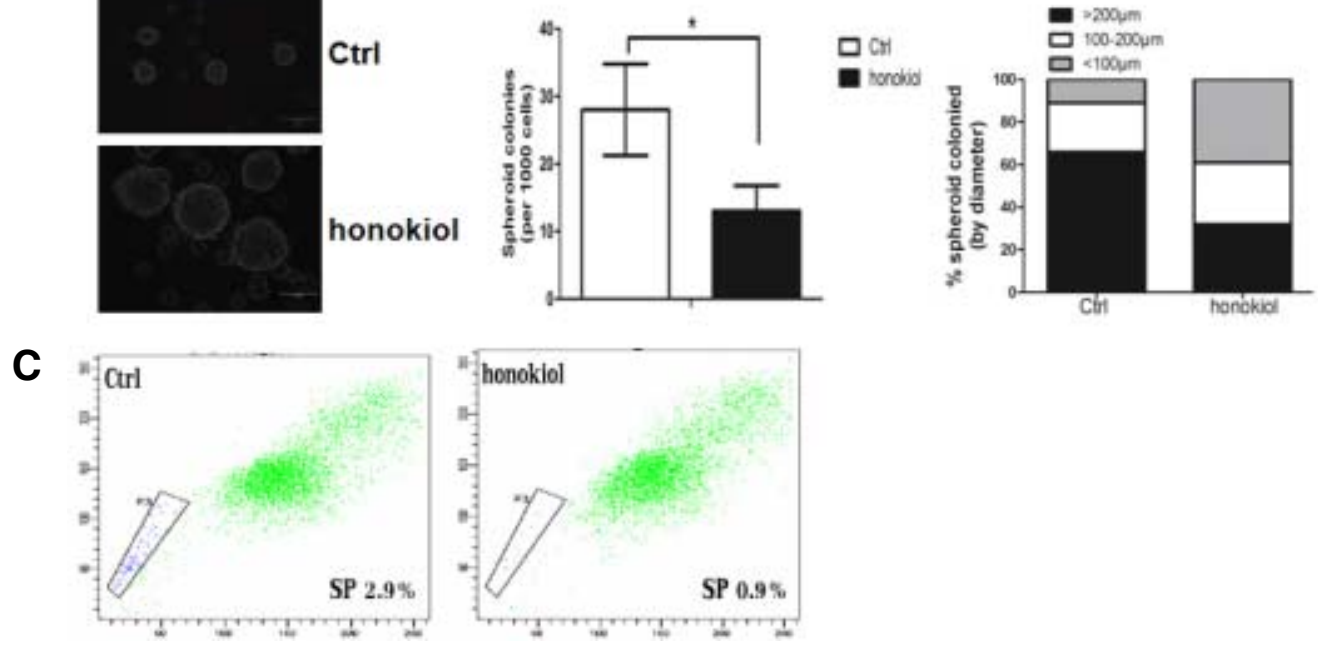

Fig. 2. Honokiol (concentration: $20 \mu \mathrm{mol}$ ) reverses EMT and suppresses CSC characteristics to inhibit cell migration and invasion. (A) Honokiol inhibited A-498 cells' migration and invasion (left panel and middle panel). Honokiol treatment altered expression level of the EMT-related proteins (right panel). (B) Honokiol inhibited the tumorsphere formation of A-498 cells with decreased number and size of sphenoid colonies. (C) Honokiol treatment suppressed the number of side population cells (SP cells), which has CSC properties, as evaluated by Hoechst 33342 staining.

of the honokiol on the viability of RCC cell line A-498. The results showed that honokiol reduced the cellular viability of A498 cells in a concentration-dependent manner (Fig. 1A). Colony formation assays showed that honokiol dramatically decreased colony formation abilities (Fig. 1B). Herein, we conclude that honokiol suppresses proliferation of RCC cells.

Honokiol reverses EMT and suppresses CSC characteristics to inhibit cell migration and invasion

We first showed that honokiol could inhibit in vitro cell migration and invasion of A-498 cells, which was accompanied by an in- crease in the expression of epithelial markers (i.e, E-cadherin) and a decrease in the expression of mesenchymal markers (i.e. fibronectin and vimentin) (Fig. 2A). Furthermore, we performed sphere formation and Hoechst 33342 exclusion assays to analyze the potential effects of honokiol on CSC characteristics in A498 cells. Indeed, honokiol significantly inhibited the tumorsphere formation of A-498 cells and decreased the number and size of sphenoid colonies (Fig. 2B). Side population cells (SP cells), with a high drug efflux capacity which was evaluated by Hoechst 33342 staining, have been suggested to possess the properties of CSC (Addla et al., 2008; Huang et al., 2013; Oates et al., 2009). 
A

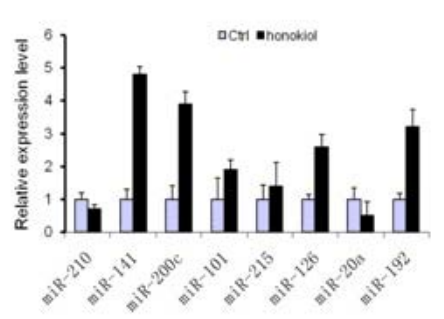

C

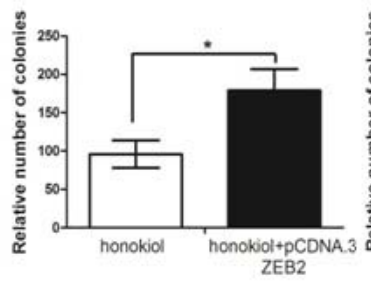
honokiol

D

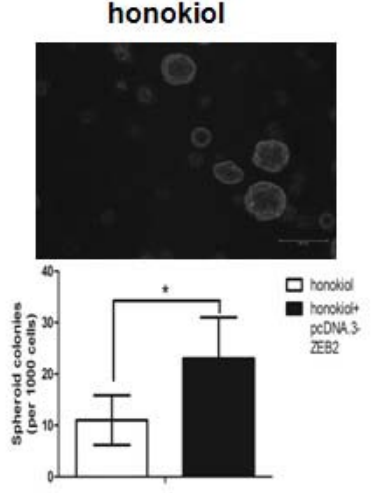

B
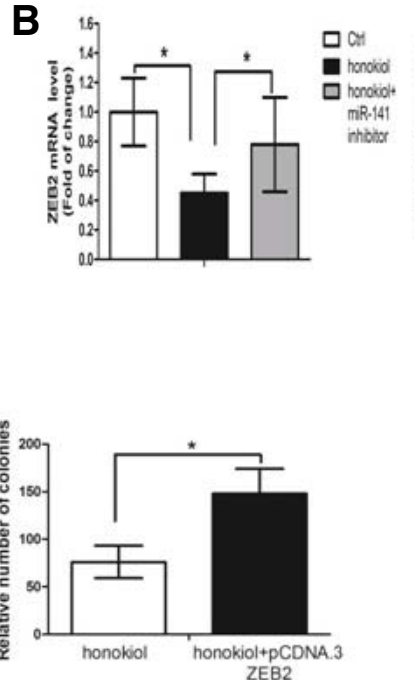

honokiol+pcDNA.3ZEB2
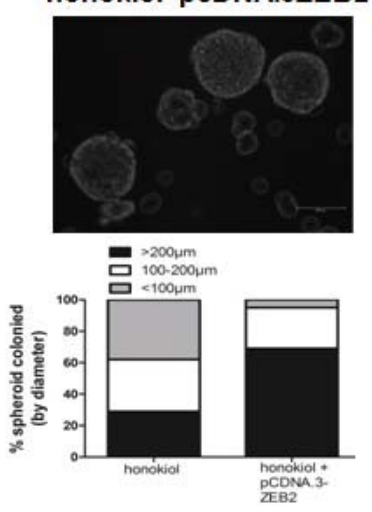
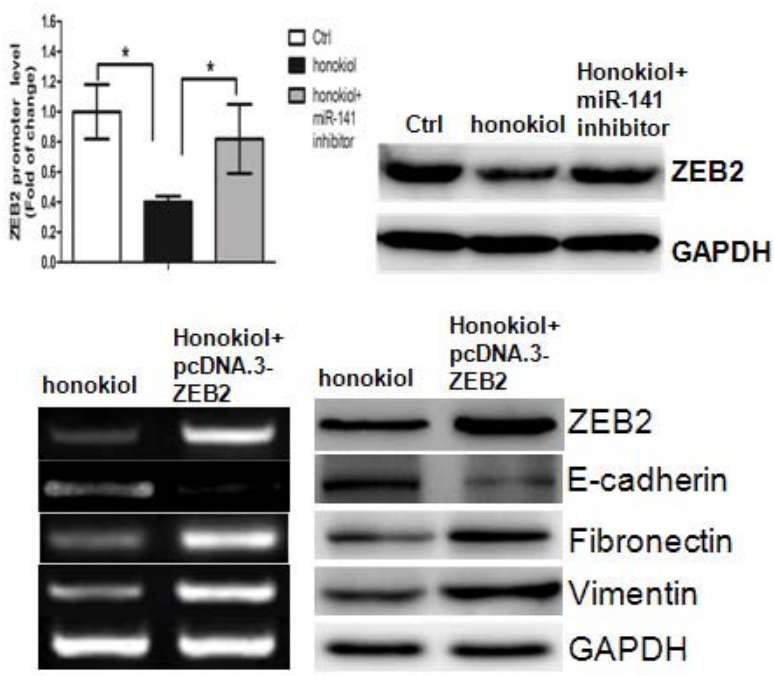

E

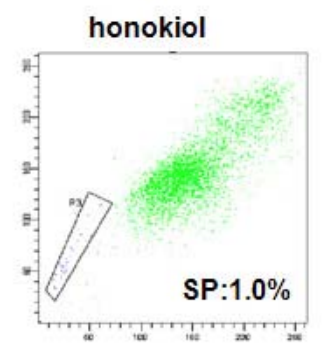

honokiol+pcDNA.3ZEB2

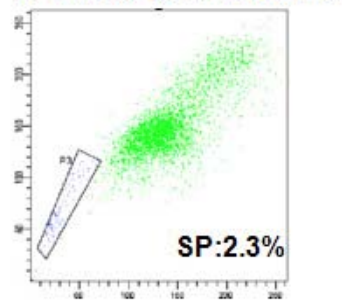

Fig. 3. Honokiol (concentration: $20 \mu \mathrm{mol}$ ) regulates EMT and CSC properties by modulating the miR-141/ZEB2 axis. (A) Changes in miRNAs expression in A-498 cells treated by honokiol, standardized to control. Mir-141 transcriptive levels notably increased approximately 4.8 -fold when compared to the control. (B) Honokiol could suppress ZEB2 expression, while a miR-141 inhibitor could partly reverse the suppressive effect of honokiol on ZEB2 expression. (C) Ectopic overexpression of ZEB2 could rescue the effect of honokiol on EMT. (D) Restoration of ZEB2 in honokiol-treated cells increased the number and size of the tumor sphere. (E) Restoration of ZEB2 in honokiol-treated cells endowed the cells with a high drug efflux capacity, as determined by Hoechst 33342 exclusion assay.

We found that honokiol decreased the number of SP cells, when compared with the control group (Fig. 2C). Taken together, these findings suggest that honokiol can target RCC cell metastasis in vitro through the reversal of EMT and the suppression of CSC properties.

The miR-141/ZEB2 axis is involved in EMT and CSC regulation by honokiol

We recently found that natural products can exert anti-tumor activities through regulating microRNAs (Deng et al., 2013; Xie et al., 2012). We thus asked whether honokiol suppressed EMT and CSC properties by modulating microRNAs. We used quantitative PCR to determine the changes of eight microRNAs which are often dysregulated in RCC (Redova et al., 2013; Wotschofsky et al., 2012). As shown in Fig. 3A, the eight miRNAs responded to honokiol treatment with different expression patterns. We focused on miR-141 which expression increased approximately 4.8-fold in A-498 cells treated with honokiol.
ZEB2, a master factor involved in EMT and CSC, has previously been identified as a target gene of miR-141 (Xu et al., 2013). We speculated that honokiol may regulate EMT and CSC properties by modulating the miR-141/ZEB2 axis. We performed qPCR, Western blot and reporter gene assays to detect the effects of honokiol on ZEB2 expression in A-498 cells. Indeed, the results showed that honokiol could suppress ZEB2 expression, while a miR-141 inhibitor could partly reverse the suppressive effect of honokiol on ZEB2 expression (Fig. 3B). We then asked whether ectopic overexpression of ZEB2 could rescue the effect of honokiol on EMT and CSC properties. As shown in Fig. 3C, the suppressive effect of honokiol on EMT can be partly rescued by ectopic overexpression of ZEB2. Interestingly, ectopic overexpression of ZEB2 increased the number and size of the tumor sphere in honokiol-treated cells (Fig. 3D). In addition, we found that the proportion of SP cells, which has CSC properties, increased significantly after overexpression of ZEB2 (Fig. 3E). 

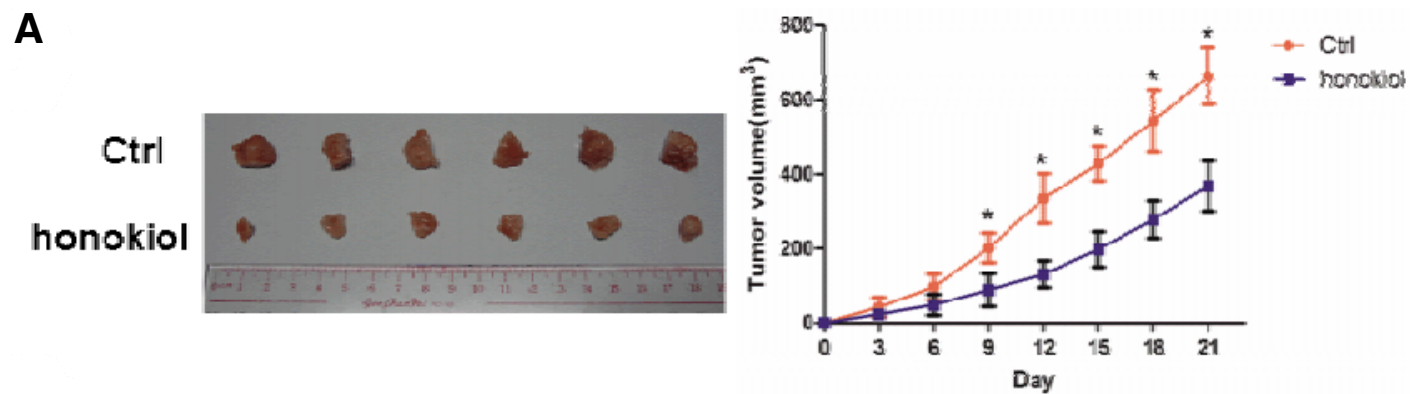

\section{ZEB2 Vimentin Fibronectin E-cadherln}

B
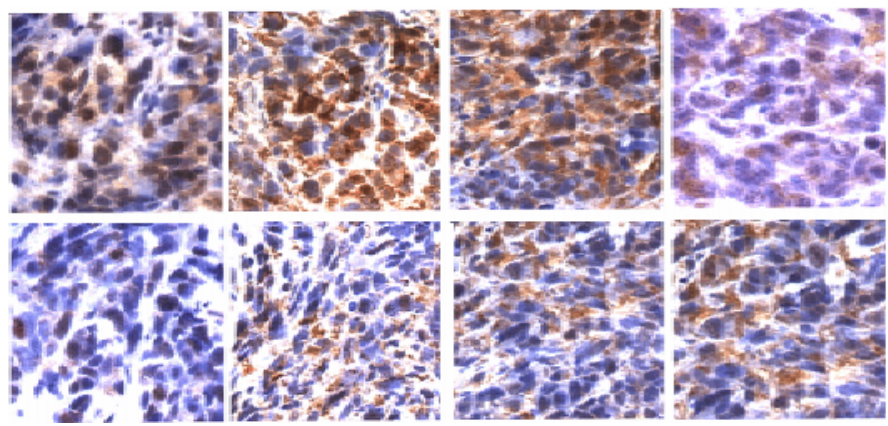

\section{CtrI}

honokiol

Fig. 4. Honokiol (concentration: $20 \mu \mathrm{mol}$ ) exhibits anti-tumor activity in A-498 xenograft tumor models. (A) The rate of tumor growth and tumor size were significantly reduced by honokiol. (B) The immunohistochemical assay showed the expression levels of ZEB2, Vimentin, Fibronectin and E-cadherin in the control group and the honokiol-treated group.

Taken together, these results demonstrate that honokiol suppresses EMT and CSC properties, at least partly, by regulating the miR-141/ZEB2 axis in RCC cells.

Honokiol exhibits anti-tumor activity in A-498 xenograft tumor models

In the experimental group treated with honokiol, the rate of tumor growth was significantly inhibited, and the tumor size was significantly reduced when compared with the control group (Fig. 4A). In addition, an immunohistochemical assay was performed to examine the level of EMT-related protein. In the group treated with honokiol the expression levels of ZEB2, Vimentin and fibronectin were decreased, while the expression level of E-cadherin was increased when compared with the control group (Fig. 4B), which was consistent with the in vitro analysis.

\section{DISCUSSION}

The antitumor activity of honokiol, a natural product derived from magnolia plant and used in traditional Chinese medicine, has been reported in various preclinical models (Fried and Arbiser, 2009). Honokiol was able to attenuate PI3K/Akt/mTOR signaling by down-regulation of Akt phosphorylation and upregulation of PTEN expression (Liu et al., 2008). In addition, honokiol was able to inhibit Ras-mediated tumor promoting pathways (Banerjee et al., 2013). Recently, researchers have reported that honokiol eliminated CSCs in some kinds of cancer. For example, honokiol targeted notch signaling to inhibit colon cancer stem cells (Ponnurangam et al., 2012). In oral cancer cells, honokiol eliminated stem-like cells and suppressed Wnt/ $\beta$-Catenin Signaling (Yao et al., 2013). However, the mole- cular mechanism how honokiol inhibits EMT and CSCs is still poorly understood.

In the current study, we investigated the potential of honokiol in the inhibition of EMT and CSC properties and the underlying molecular mechanisms. The following novel findings are reported in this study: (i) honokiol treatment inhibits malignant properties such as invasion and migration of RCC cells; (ii) honokiol suppresses renal cancer cells' metastasis dualblocking epithelial-mesenchymal transition and cancer stem cell properties; (iii) Regulation of EMT and CSC properties by honokiol requires the miR-141/ZEB2 axis.

MiR-141 is a putative tumor-suppressive miRNAs which is downregulated in RCC (Nakada et al., 2008; Yoshino et al., 2013). The relation between miR-141 and honokiol has not been reported before. We demonstrated that honokiol can upregulate the expression level of miR-141. It may be possible that honokiol elevates miR-141 expression through inhibiting histone deacetylases (Singh et al., 2013), which are suggested to be responsible for the downregulation of miR-141 (Roy et al., 2013). ZEB2, a target of miR-141 (Xu et al., 2013), is a master factor involved in EMT and CSC (Kong et al., 2011) and is frequently overexpressed in RCC (Fang et al., 2013; Henrion et al., 2013). We found that honokiol exerted anti-tumor activities through modulating the miR-141/ZEB2 axis, and that ectopic overexpression of ZEB2 could rescue the effect of honokiol on EMT and CSC properties. It will be important to determine whether our findings are widespread in various cancer cells or cell-type-specific.

In summary, our results showed that honokiol treatment significantly inhibits malignant properties of RCC cells through modulation of EMT and CSC properties; thus using honokiol may be a suitable therapeutic strategy for RCC treatment. 


\section{ACKNOWLEDGMENTS}

This work was supported by Research Project of Guangzhou Medical University (2013C61).

\section{REFERENCES}

Addla, S.K., Brown, M.D., Hart, C.A., Ramani, V.A., and Clarke, N.W. (2008). Characterization of the Hoechst 33342 side population from normal and malignant human renal epithelial cells. Am. J. Physiol. Renal Physiol. 295, F680-687.

Ambros, V. (2004). The functions of animal microRNAs. Nature 431, 350-355.

Banerjee, P., Basu, A., Arbiser, J.L., and Pal, S. (2013). The natural product honokiol inhibits calcineurin inhibitor-induced and Rasmediated tumor promoting pathways. Cancer Lett. 338, 292-299.

Cheng, L., Zhang, S., MacLennan, G.T., Lopez-Beltran, A., and Montironi, R. (2009). Molecular and cytogenetic insights into the pathogenesis, classification, differential diagnosis, and prognosis of renal epithelial neoplasms. Hum. Pathol. 40,10-29.

Croce, C.M., and Calin, G.A. (2005). miRNAs, cancer, and stem cell division. Cell 122, 6-7.

Deng, X., Ma, L., Wu, M., Zhang, G., Jin, C., Guo, Y., and Liu, R. (2013). miR-124 radiosensitizes human glioma cells by targeting CDK4. J. Neurooncol. 114, 263-274.

Fang, Y., Wei, J., Cao, J., Zhao, H., Liao, B., Qiu, S., Wang, D., Luo, J., and Chen, W. (2013). Protein expression of ZEB2 in renal cell carcinoma and its prognostic significance in patient survival. PLoS One 8, e62558.

Fried, L.E., and Arbiser, J.L. (2009). Honokiol, a multifunctional antiangiogenic and antitumor agent. Antioxid. Redox Signal. 11, 1139-1148.

Gregory, P.A., Bracken, C.P., Bert, A.G., and Goodall, G.J. (2008). MicroRNAs as regulators of epithelial-mesenchymal transition Cell Cycle 7, 3112-3118.

Gupta, P.B., Onder, T.T., Jiang, G., Tao, K., Kuperwasser, C., Weinberg, R.A., and Lander, E.S. (2009). Identification of selective inhibitors of cancer stem cells by high-throughput screening. Cell 138, 645-659.

Henrion, M., Frampton, M., Scelo, G., Purdue, M., Ye, Y., Broderick, P., Ritchie, A., Kaplan, R., Meade, A., McKay, J., et al. (2013). Common variation at 2q22.3 (ZEB2) influences the risk of rena cancer. Hum. Mol. Genet. 22, 825-831.

Huang, B., Huang, Y.J., Yao, Z.J., Chen, X., Guo, S.J., Mao, X.P., Wang, D.H., Chen, J.X., and Qiu, S.P. (2013). Cancer stem celllike side population cells in clear cell renal cell carcinoma cell line 769P. PLoS One 8, e68293.

Kong, D., Li, Y., Wang, Z., and Sarkar, F.H. (2011). Cancer stem cells and epithelial-to-mesenchymal transition (EMT)-phenotypic cells: are they cousins or twins? Cancers 3, 716-729.

Kumar, A., Kumar Singh, U., and Chaudhary, A. (2013). Honokiol analogs: a novel class of anticancer agents targeting cell signaling pathways and other bioactivities. Future Med. Chem. 5, 809829.

Lee, Y.J., Lee, Y.M., Lee, C.K., Jung, J.K., Han, S.B., and Hong, J.T. (2011). Therapeutic applications of compounds in the Magnolia family. Pharmacol. Ther. 130, 157-176.

Liu, H., Zang, C., Emde, A., Planas-Silva, M.D., Rosche, M., Kuhnl, A., Schulz, C.O., Elstner, E., Possinger, K., and Eucker, J. (2008). Anti-tumor effect of honokiol alone and in combination with other anti-cancer agents in breast cancer. Eur. J. Pharmacol. 591, 43-51.

Ma, L., Zhang, G., Miao, X.B., Deng, X.B., Wu, Y., Liu, Y., Jin, Z.R., Li, X.Q., Liu, Q.Z., Sun, D.X., et al. (2013). Cancer stem-like cell properties are regulated by EGFR/AKT/beta-catenin signaling and preferentially inhibited by gefitinib in nasopharyngeal carcinoma. FEBS J. 280, 2027-2041.

Mani, S.A., Guo, W., Liao, M.J., Eaton, E.N., Ayyanan, A., Zhou, A.Y., Brooks, M., Reinhard, F., Zhang, C.C., Shipitsin, M., et al. (2008). The epithelial-mesenchymal transition generates cells with properties of stem cells. Cell 133, 704-715.

McLaughlin, J.K., Lipworth, L., and Tarone, R.E. (2006). Epidemiologic aspects of renal cell carcinoma. Semin. Oncol. 33, 527533.
Motzer, R.J., Mazumdar, M., Bacik, J., Berg, W., Amsterdam, A. and Ferrara, J. (1999). Survival and prognostic stratification of 670 patients with advanced renal cell carcinoma. J. Clin. Oncol. 17, 2530-2540.

Moutasim, K.A., Nystrom, M.L., and Thomas, G.J. (2011). Cell migration and invasion assays. Methods Mol. Biol. 731, 333-343.

Nakada, C., Matsuura, K., Tsukamoto, Y., Tanigawa, M., Yoshimoto, T., Narimatsu, T., Nguyen, L.T., Hijiya, N., Uchida, T., Sato, F., et al. (2008). Genome-wide microRNA expression profiling in renal cell carcinoma: significant down-regulation of miR-141 and miR-200c. J. Pathol. 216, 418-427.

Newman, D.J., Cragg, G.M., and Snader, K.M. (2003). Natural products as sources of new drugs over the period 1981-2002. J. Nat. Products 66, 1022-1037.

Oates, J.E., Grey, B.R., Addla, S.K., Samuel, J.D., Hart, C.A., Ramani, V.A., Brown, M.D., and Clarke, N.W. (2009). Hoechst 33342 side population identification is a conserved and unified mechanism in urological cancers. Stem Cells Dev. 18, 15151522.

Polyak, K., and Weinberg, R.A. (2009). Transitions between epithelial and mesenchymal states: acquisition of malignant and stem cell traits. Nat. Rev. Cancer 9, 265-273.

Ponnurangam, S., Mammen, J.M., Ramalingam, S., He, Z., Zhang, Y., Umar, S. Subramaniam, D., and Anant, S. (2012). Honokiol in combination with radiation targets notch signaling to inhibit coIon cancer stem cells. Mol. Cancer Ther. 11, 963-972.

Radisky, D.C., and LaBarge, M.A. (2008). Epithelial-mesenchymal transition and the stem cell phenotype. Cell Stem Cell 2, 511512.

Redova, M., Poprach, A., Besse, A., lliev, R., Nekvindova, J., Lakomy, R., Radova, L., Svoboda, M., Dolezel, J., Vyzula, R., et al. (2013). MiR-210 expression in tumor tissue and in vitro effects of its silencing in renal cell carcinoma. Tumour Biol. 34, 481-491.

Roy, S.S., Gonugunta, V.K., Bandyopadhyay, A., Rao, M.K., Goodall, G.J., Sun, L.Z., Tekmal, R.R., and Vadlamudi, R.K. (2013). Significance of PELP1/HDAC2/miR-200 regulatory network in EMT and metastasis of breast cancer. Oncogene [Epub ahead of print].

Singh, T., Prasad, R., and Katiyar, S.K. (2013). Inhibition of class I histone deacetylases in non-small cell lung cancer by honokiol leads to suppression of cancer cell growth and induction of cell death in vitro and in vivo. Epigenetics 8, 54-65.

Taube, J.H., Malouf, G.G., Lu, E., Sphyris, N., Vijay, V., Ramachandran, P.P., Ueno, K.R., Gaur, S., Nicoloso, M.S., Rossi, S., et al. (2013). Epigenetic silencing of microRNA-203 is required for EMT and cancer stem cell properties. Sci. Rep. 3, 2687.

Tian, W., Xu, D., and Deng, Y.C. (2012). Honokiol, a multifunctional tumor cell death inducer. Die Pharmazie 67, 811-816.

Valster, A., Tran, N.L., Nakada, M., Berens, M.E., Chan, A.Y., and Symons, M. (2005). Cell migration and invasion assays. Methods 37, 208-215.

Wotschofsky, Z., Liep, J., Meyer, H.A., Jung, M., Wagner, I., Disch, A.C., Schaser, K.D., Melcher, I., Kilic, E., Busch, J., et al. (2012) Identification of metastamirs as metastasis-associated microRNAs in clear cell renal cell carcinomas. Int. J. Biol. Sci. 8, 13631374.

Xie, Y.K., Huo, S.F., Zhang, G., Zhang, F., Lian, Z.P., Tang, X.L., and Jin, C. (2012). CDA-2 induces cell differentiation through suppressing Twist/SLUG signaling via miR-124 in glioma. J. Neurooncol. 110, 179-186.

Xu, H., Mei, Q., Xiong, C., and Zhao, J. (2013). Tumor-suppressing effects of miR-141 in human osteosarcoma. Cell Biochem. Biophys. [Epub ahead of print].

Yao, C.J., Lai, G.M., Yeh, C.T., Lai, M.T., Shih, P.H., Chao, W.J., Whang-Peng, J., Chuang, S.E., and Lai, T.Y. (2013). Honokiol eliminates human oral cancer stem-like cells accompanied with suppression of Wnt/ beta -catenin signaling and apoptosis induction. Evid. Based Complement. Alternat. Med. 2013, 146136.

Yoshino, H., Enokida, H., Itesako, T., Tatarano, S., Kinoshita, T., Fuse, M., Kojima, S., Nakagawa, M., and Seki, N. (2013). Epithelial-mesenchymal transition-related microRNA-200s regulate molecular targets and pathways in renal cell carcinoma. J. Hum. Genet. 58, 508-516. 